\title{
Review of top quark production at LHC and Tevatron
}

\author{
Kentaro KAWADE* \\ Nagoya University, Japan \\ On behalf of ATLAS, CDF, CMS, DØ, and LHCb collaborations \\ E-mail: kawade@hepl.phys.nagoya-u.ac.jp
}

\begin{abstract}
A summary of recent results on top quark production at LHC and Tevatron are presented in this paper. The measurements of top quark production were performed in various energies $(\sqrt{s}=$ $1.96,7,8,13 \mathrm{TeV}$ ) and initial states ( $p \bar{p}$ and $p p$ ) by the ATLAS, CDF, CMS, D $\emptyset$ and LHCb experiments. The measured top quark pair production cross-section is in good agreement with the standard model predictions in each energy region. Differential cross-sections were also measured and compared with several theoretical predictions. Most of the higher order QCD models reproduce the experimental data well, while a slight discrepancy in a certain phase space such as higher top quark $p_{T}$ region is observed. Electroweak single top quark production was also measured and the results are consistent with theoretical predictions at the various energies.
\end{abstract}

XIII International Conference on Heavy Quarks and Leptons

22-27 May, 2016

Blacksburg, Virginia, USA

${ }^{*}$ Speaker. 


\section{Introduction}

The top quark is the heaviest elementary particle in the standard model and was discovered in 1995 by the CDF and DØ experiments at Tevatron [1,2]. The top quark mass of $173 \mathrm{GeV}$ indicates strong Higgs-Yukawa coupling, $y_{t} \simeq 1$. It decays within $10^{-25}$ seconds as "bare-quark" before hadronization. Therefore, the top quark plays a special role in electroweak symmetry breaking.

The study of the production of the top quark is important to understand the standard model QCD and EW processes and the top quark production is sensitive to physics beyond the standard model. In hadron colliders, the top quarks are produced as top quark and anti-top quark pair via QCD interaction, or singly via EW interaction. At Tevatron $85 \%$ of the top quark pairs are produced by $q \bar{q}$ annihilation processes, while at the LHC $90 \%$ of them are produced by $g g$ fusion processes. The single top produced by three different types of productions called $t$-channel, $s$-channel and $W t$-channel.

The top quark decays to a $W$ boson and a bottom quark with almost $100 \%$ branching ratio. The signature of top quark events is characterized by high $p_{T}$ isolated leptons or jets from the $W$ boson decay, $b$-jets originating from the decay of bottom quark, and large missing transverse momentum due to the presence of neutrinos.

\section{Top quark pair production cross-section}

The CDF and DØ experiments measured the top quark pair production cross-section at $\sqrt{s}=$ $1.96 \mathrm{TeV}$ in various final states and with different methods. The combined cross section is $\sigma_{t \bar{t}}=$ $7.60 \pm 0.41 \mathrm{pb}$ for a top-quark mass of $m_{t}=172.5 \mathrm{GeV}$ [3]. Figure 1 shows the results of $\sigma_{t \bar{t}}$ measurements from the $\mathrm{CDF}$ and $\mathrm{D} \emptyset$ experiments and their combination.

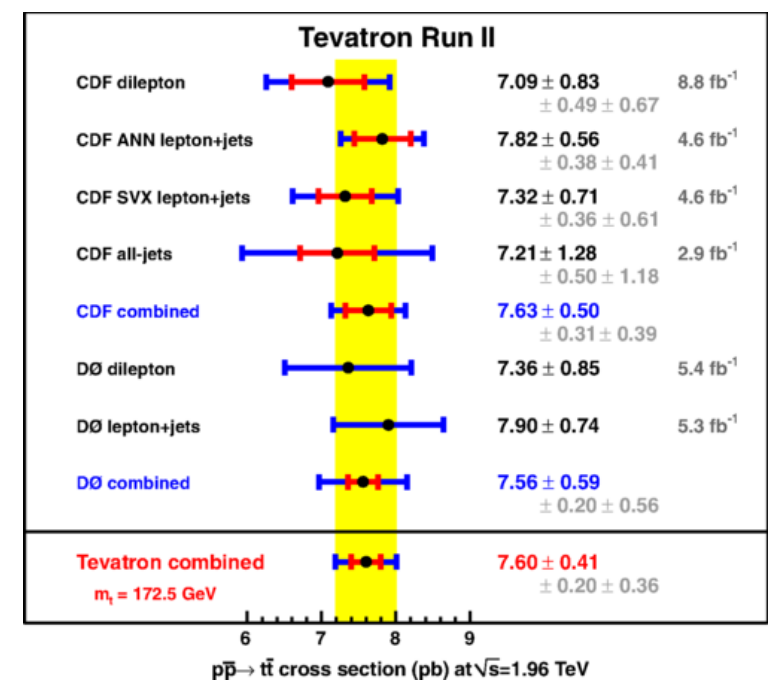

Figure 1: The results of $\sigma_{t \bar{t}}$ measurement from the CDF and $\mathrm{D} \emptyset$ experiments and their combination [3]. The inner and outer error bars show statistical and total uncertainties, respectively.

DØ recently measured the top quark pair cross-section in the lepton+jets and dilepton final states with the full data-set corresponding to $9.7 \mathrm{fb}^{-1}$ taken during the Tevatron Run II period. Their 
own combined inclusive cross-section is measured to be $\sigma_{t \bar{t}}=7.73 \pm 0.13$ (stat) \pm 0.55 (syst) pb [4], where the relative total uncertainty is $7.3 \%$. These results are consistent with the standard model prediction. They also extracted a pole mass of the top quark to be $m_{t}=169.5_{-3.4}^{+3.3} \mathrm{GeV}$, which is the most precise top quark pole mass measurement at Tevatron.

The CMS [5] and ATLAS [6] experiments have measured the top quark pair production crosssection at $\sqrt{s}=13 \mathrm{TeV}$ using the very clean signature with the dilepton $e \mu$ final state. CMS obtained an inclusive cross-section of $\sigma_{t \bar{t}}=793 \pm 8$ (stat) \pm 38 (syst) \pm 21 (lumi) pb, using the dataset corresponding to an integrated luminosity of $2.2 \mathrm{fb}^{-1}$ [7]. ATLAS also measured the inclusive cross-section to be $\sigma_{t \bar{t}}=818 \pm 8$ (stat) \pm 27 (syst) \pm 19 (lumi) \pm 12 (beam) pb, using the data-set corresponding to an integrated luminosity of $3.2 \mathrm{fb}^{-1}$ [8].

Figure 2 shows the summary of the top quark pair production cross-section measurements by the LHC and Tevatron experiments as a function of the centre-of-mass energy [9]. The experimental results are consistent with the next-to-next leading order (NNLO) QCD calculation complemented with next-to-next leading logarithm resummation (NNLL).

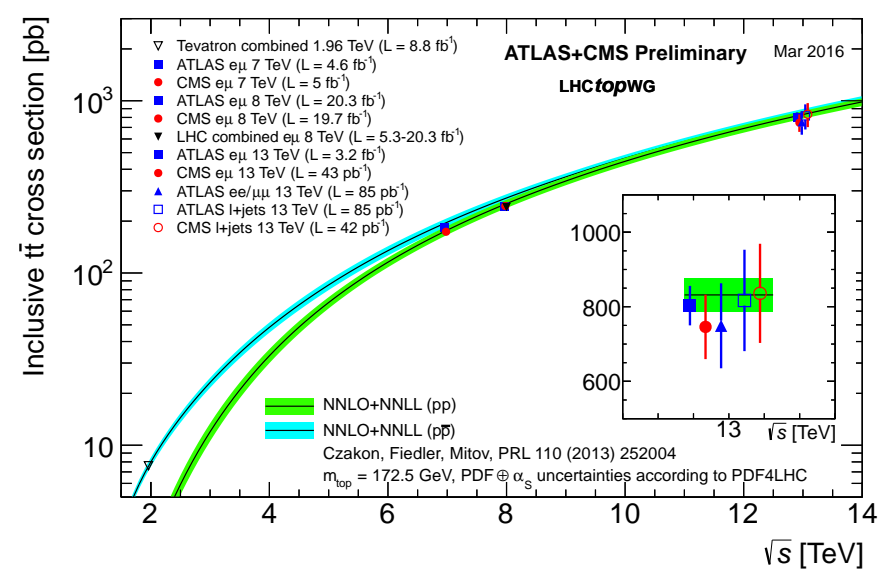

Figure 2: Summary of the top quark pair production cross-section measurements by LHC and Tevatron experiments as a function of the centre-of-mass energy compared to the NNLO QCD calculation complemented with NNLL resummation [9]. The error bands for theory show the uncertainties due to renormalization and factorization scale, PDFs and the strong coupling constant.

ATLAS measured the ratio of the $t \bar{t}$ and Z-boson production cross-sections $\left(R_{t \bar{t} / Z}\right)$ as shown in Figure 3, based on the measurement of the $t \bar{t}$ cross-section in the dilepton $e \mu$ final state and the measurement of the $Z$-boson cross-section in the $e e$ and $\mu \mu$ final states [11]. This ratio benefits from significant cancellations in several systematic uncertainties from integrated luminosity and the lepton reconstruction. The ratio is sensitive to ratios of the gluon and sea-quark Parton distribution Function (PDF).

\section{Top quark pair differential cross-section}

Differential distribution as a function of the top quark kinematic variables and the activity of additional jets provides a more precise test of the standard model. The transverse momentum, 


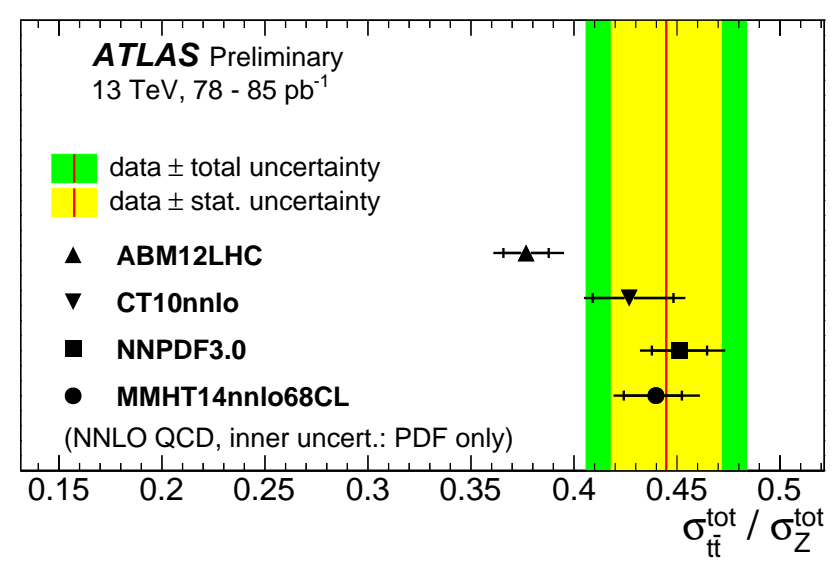

Figure 3: The cross-section ratio $R_{t \bar{t} / Z}$ with NNLO predictions at $\sqrt{s}=13 \mathrm{TeV}$ based on the ABM12LHC, CT10, NNPDF3.0 and MMHT14 PDF sets [11].

the rapidity, and the invariant mass of the top quark pair system are sensitive to the level of the initial and final state radiations, PDFs, and the contributions from physics beyond the standard model, respectively. The activity of additional jets is a good probe for higher order QCD effects, in particular matching between the matrix-element generator and the shower model. Thus, it is a quite interesting program at the hadron colliders to check various distributions in top quark events thoroughly.

$\mathrm{D} \emptyset$ measured the differential cross-section as a function of the invariant mass, the transverse momentum and the absolute value of the rapidity of the top quark pair system in $p \bar{p}$ collisions at $\sqrt{s}=1.96 \mathrm{TeV}$ [12]. Figure 4 shows the measured differential cross section as a function of the invariant mass of the top quark pair system together with several QCD predictions. There is no evidence for new physics and the results are consistent with the standard model predictions within the uncertainties.
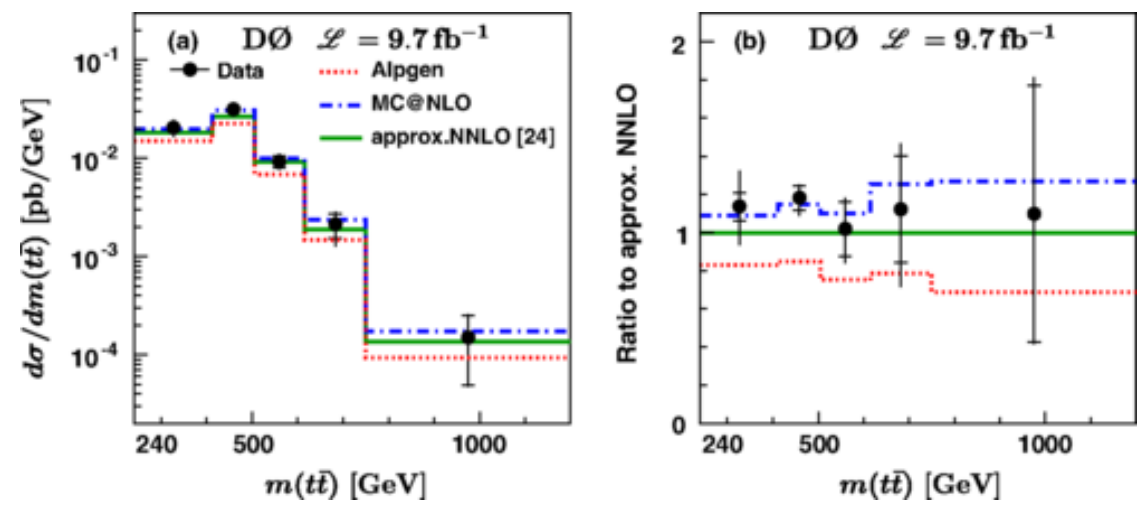

Figure 4: The left panel shows the measured differential cross section as a function of the invariant mass of the top quark pair system together with several QCD predictions [12]. The inner error bars correspond to the statistical uncertainties and the outer error bars correspond to the total uncertainties. The right panel shows the ratio of data to the Monte Carlo (MC) predictions and the QCD prediction at approximate NNLO.

CDF reported a measurement of the differential top-quark pair production cross-section as a 
function of the top-quark production angle $\theta_{t}$ in $p \bar{p}$ collisions at $\sqrt{s}=1.96 \mathrm{TeV}$ [13]. Figure 5 show fraction of cross-section accruing in 10 bins of $\cos \theta_{t}$, obtained by integrating the series of Legendre polynomials over the width of each bin. The observed linear-term coefficient of the $\cos \theta_{t}$ of $a_{1}=0.39 \pm 0.11$ is in excess of the NLO SM prediction of $a_{1}=0.15_{-0.03}^{+0.07}$, but the NLO SM prediction agree with $\cos \theta_{t}$ distribution within the uncertainties.

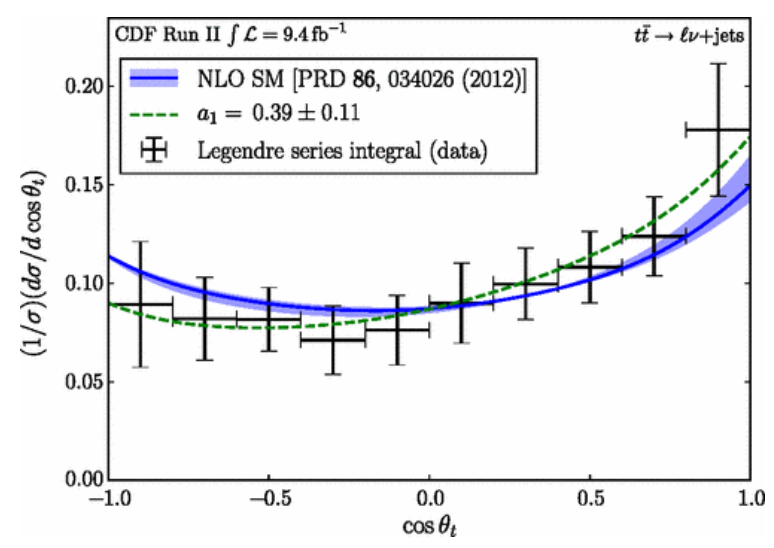

Figure 5: Fraction of cross-section accruing in 10 bins of $\cos \theta_{t}$, obtained by integrating the series of Legendre polynomials over the width of each bin [13].

Measurements of the normalized differential top quark pair production cross-section as a function of the multiplicity of additional jets were performed using $3.2 \mathrm{fb}^{-1}$ of $p p$ collision data at $\sqrt{s}=$ $13 \mathrm{TeV}$ by ATLAS [14]. The measured data were unfolded to particle-level in the fiducial phase space. The multiplicity of additional jets was obtained for various jet $p_{T}$ thresholds of 25, 40, 60 and $80 \mathrm{GeV}$. Figure 6 shows the unfolded distribution of the multiplicity of additional jets with $p_{T}$ $>25 \mathrm{GeV}$ in the $e \mu$ final state. The results were compared to several NLO MC predictions and found to be in good agreement with the predictions.
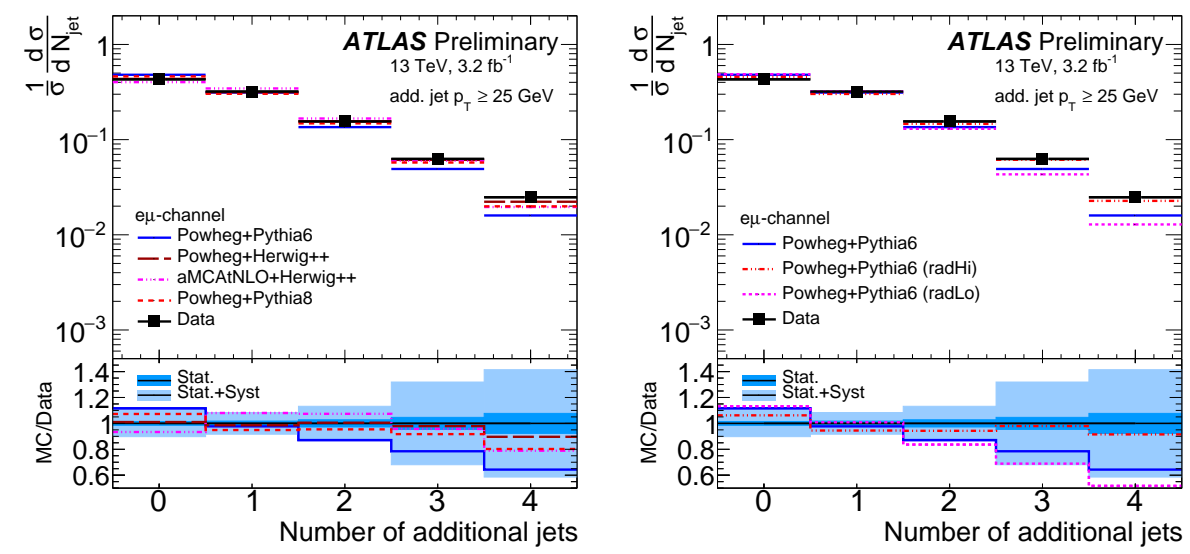

Figure 6: Unfolded distribution of the multiplicity of additional jets with $p_{T}>25 \mathrm{GeV}$ in the $e \mu$ final state [14]. The results are compared with different MC generator predictions (left) and the Powheg+Pythia6 predictions with various QCD radiation scale (right). The bottom panels show the ratio of predictions to the data. 
CMS measured the normalized differential $t \bar{t}$ production cross-section in $p p$ collisions at $\sqrt{s}=$ $13 \mathrm{TeV}$ in the $\ell+$ jets [15] and dilepton final states [16]. Figure 7 shows the normalized differential cross-section as a function of the top quark $p_{T}$ compared to the various $\mathrm{MC}$ predictions in the $\ell+$ jets final state. The left and right panels in Figure 7 correspond to the hadronic and leptonic decaying top quarks, respectively. The results are consistent with the MC predictions within un-
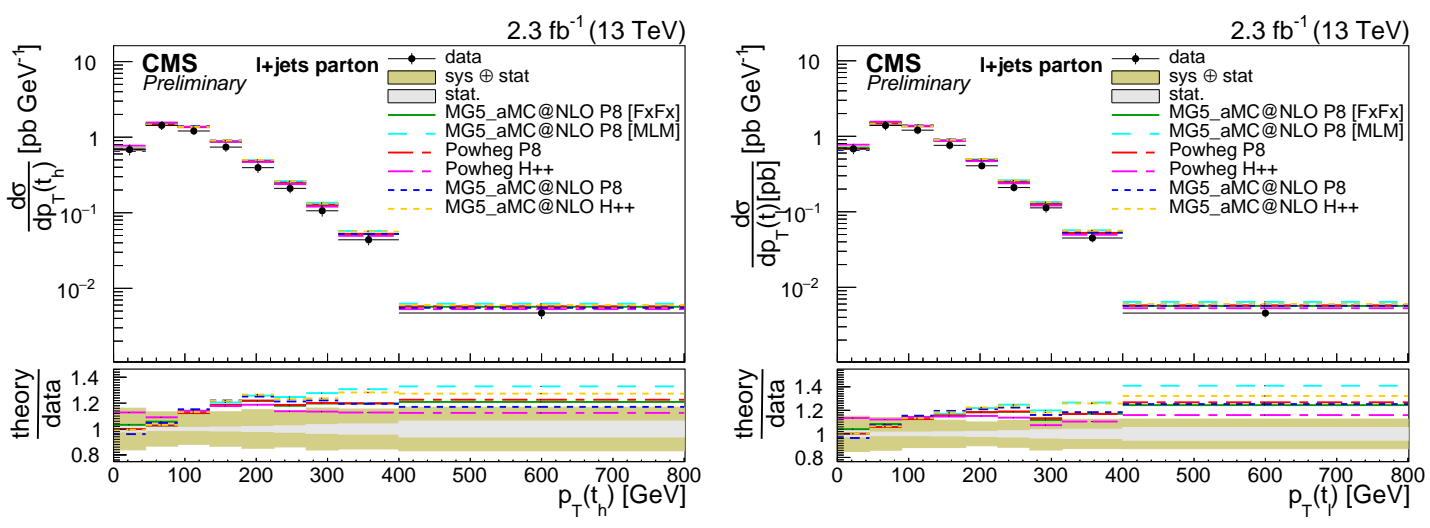

Figure 7: Normalized differential cross-sections as a function of the top quark $p_{T}$ compared to the predictions of Powheg+Pythia8, Powheg+Herwig++, MG5-aMC@NLO+Pythia8, and MadGRAPH+PYthiA8 [15]. The left and right panels correspond to the hadronic and leptonic decaying top quark, respectively. The bottom panels show the ratio of predictions to the data.

certainties, while data show a slightly softer spectrum than MC in the higher top quark $p_{T}$ region. Figure 8 shows the normalized differential cross-section as a function of the top quark $p_{T}$ and of the additional jet multiplicity measured in the dilepton final state. The measured top quark $p_{T}$ spectrum is found to be softer than MC predictions as in the $\ell+$ jets final state. The higher order QCD calculations describe data better.

Figure 9 shows the measured full phase-space normalized differential $t \bar{t}$ cross-section as a function of the transverse momentum of the top quark measured by ATLAS [17] and CMS [18] at $\sqrt{s}=8 \mathrm{TeV}$ together with NNLO and approximate next-to-next-to-next leading order (NNNLO) calculations. The higher order QCD calculations show better agreement with the data.

\section{Observation of the top quark production in the forward region}

The LHCb [19] experiment published a measurement of the top quark production in the forward region of $p p$ collision for $\sqrt{s}=7$ and $8 \mathrm{TeV}$, where the $t \bar{t}$ production via $q \bar{q}$ annihilation is enriched. The forward top quark production is also sensitive to the high- $x$ gluon PDF. In the forward region, $75 \%$ of the top quarks are produced in $t \bar{t}$ pair production, while $25 \%$ of the top quarks are produced in single top production, including mainly $t$-channel process and a few percent of $s$-channel and $W t$-channel. In this measurement, a forward muon with a transverse momentum $p_{T}$ larger than $25 \mathrm{GeV}$ in the pseudo-rapidity range $2.0<\eta<4.5$ and a bjet with $50 \mathrm{GeV}<p_{T}<100 \mathrm{GeV}$ and $2.2<\eta<4.2$ are required. The measured cross-sections are $\sigma_{\text {top }}=239 \pm 53$ (stat) \pm 33 (syst) \pm 24 (theory) fb at $\sqrt{s}=7 \mathrm{TeV}$, and $\sigma_{\text {top }}=289 \pm 43$ (stat) \pm 40 (syst) \pm 29 (theory) fb at $\sqrt{s}=8 \mathrm{TeV}$ [20]. These results are in agreement with NLO standard model predictions. 

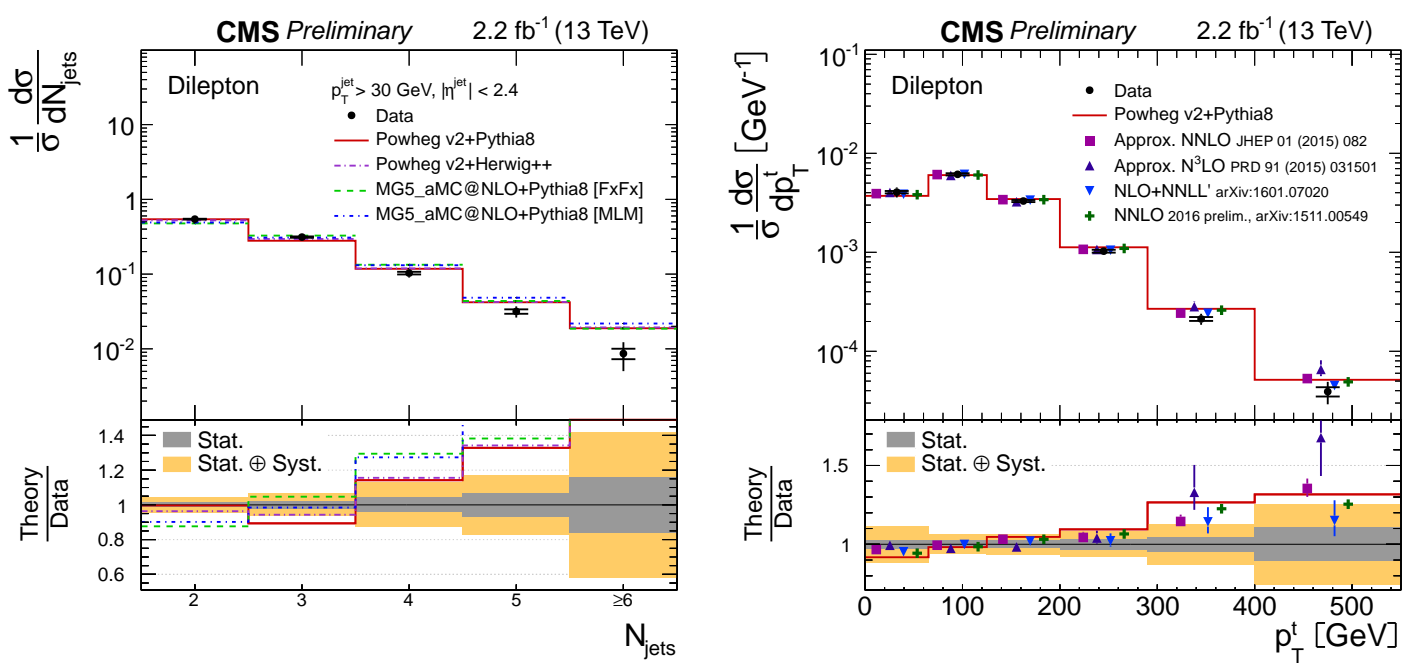

Figure 8: Normalized differential $t \bar{t}$ cross-sections as a function of the jet multiplicity (left) and the top quark $p_{T}$ (right) [16]. The data are compared to various MC predictions and several beyond-NLO QCD calculations. The bottom panels show the ratio of predictions to the data.

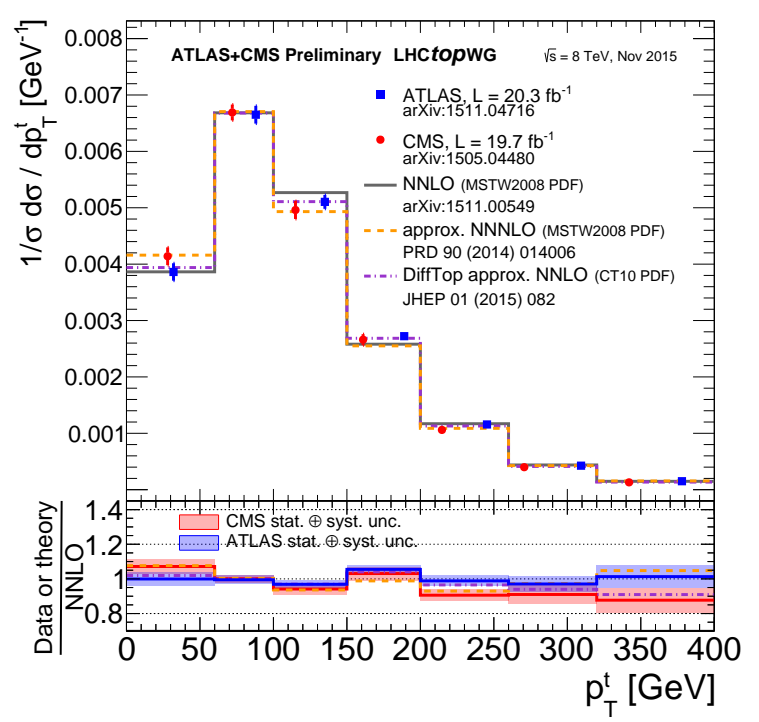

Figure 9: Measured full phase-space normalized differential $t \bar{t}$ cross-section as a function of the transverse momentum of the top quark together with NNLO and approximate NNLO calculations [17, 18] [9]. The lower panel shows the ratio of the data the approximate NNLO calculations to the full NNLO calculation. 


\section{Single top production cross-section}

The single top quark production cross-section is expected to be proportional to $\left|V_{t b}\right|^{2}$, where $V_{t b}$ is the element of the Cabibbo-Kobayashi-Maskawa matrix. It is sensitive to contributions from fourth generation quarks, as well as flavour-changing neutral current processes, heavy $W^{\prime}$ bosons, and $H^{ \pm}$bosons. Understanding of the single top quark production is also important to estimate the number of SM backgrounds more precisely for the BSM searches.

\section{$5.1 s$-channel single top production}

The first observation of single top production in the $s$-channel is reported through the combination of the $\mathrm{CDF}$ and $\mathrm{D} \emptyset$ measurements for $p \bar{p}$ collisions at $\sqrt{s}=1.96 \mathrm{TeV}$ [21]. The data correspond to a total integrated luminosity of up to $9.7 \mathrm{fb}^{-1}$ for each experiment. The $s$-channel single top events were selected by requiring a high $p_{T}$ lepton, large missing transverse momentum, and one or two $b$-jets. The combined cross-section is measured to be $\sigma_{s-\mathrm{ch}}=1.29_{-0.24}^{+0.26} \mathrm{pb}$ with a significance of $6.3 \sigma$.

ATLAS reported evidence for single top-quark production in the $s$-channel using $p p$ collisions at $\sqrt{s}=8 \mathrm{TeV}$ [22]. The signal is extracted using a maximum-likelihood fit to a discriminant which is based on the matrix element method for the separation from the backgrounds of $t \bar{t}$ production and $W$-boson production in association with heavy-flavour jets. The cross-section is measured to be $\sigma_{s-\mathrm{ch}}=4.8 \pm 0.8(\mathrm{stat})_{-1.3}^{+1.6}$ (syst) pb with a significance of $3.2 \sigma$, and is consistent with the SM prediction. CMS searched for $s$-channel single top production using data of in $p p$ collisions at $\sqrt{s}=7$ and $8 \mathrm{TeV}$ [23]. The signal is extracted using a maximum-likelihood fit to a multivariate discriminant obtained using a Boosted Decision Tree (BDT). The measured cross-sections are $\sigma_{s-\mathrm{ch}}=7.1 \pm 8.1 \mathrm{pb}$ for $7 \mathrm{TeV}$ and $\sigma_{s-\mathrm{ch}}=13.4 \pm 7.3 \mathrm{pb}$ for $8 \mathrm{TeV}$.

\section{$5.2 t$-channel single top production}

ATLAS and CMS performed the measurement of $t$-channel single top production cross-section in $p p$ collisions at $\sqrt{s}=13 \mathrm{TeV}[24,25]$. Both measurements used a binned maximum-likelihood fit to the distribution of the output of a neural network. The cross-sections are determined to be $\sigma(t q)=133 \pm 6$ (stat.) \pm 24 (syst.) \pm 7 (lumi.) pb for single top-quark production and $\sigma(\bar{q} q)=$ $96 \pm 5$ (stat.) \pm 23 (syst.) \pm 5 (lumi.) pb for single anti-top quark production by ATLAS. CMS measured the cross-section to be $\sigma(t q)=141.5 \pm 6.7$ (stat. $) \pm 9.4$ (exp. $)_{-19.6}^{+19.3}$ (theo.) \pm 3.8 (lumi.) pb and $\sigma(\bar{t} q)=81.0 \pm 6.2$ (stat.) \pm 8.1 (exp. $)_{-10.9}^{+10.9}$ (theo.) \pm 2.2 (lumi.) pb.

CMS also measured the differential $t$-channel single top cross-section as a function of $p_{T}$ and the rapidity of the top quark [26]. The differential measurement is particularly well suited to the test of the modeling of single top production. Figure 10 shows the differential cross-sections at parton level in full phase-space. There is no significant deviation from the MC predictions within the experimental uncertainties.

\subsection{Wt-channel single top production}

The cross-section for the single top quark production in association with a $W$ boson in $p p$ collisions at $\sqrt{s}=8 \mathrm{TeV}$ was measured by ATLAS [27] selecting events containing two leptons and one central $b$-jet. The $W t$ signal is separated from the backgrounds using a multivariate analysis 

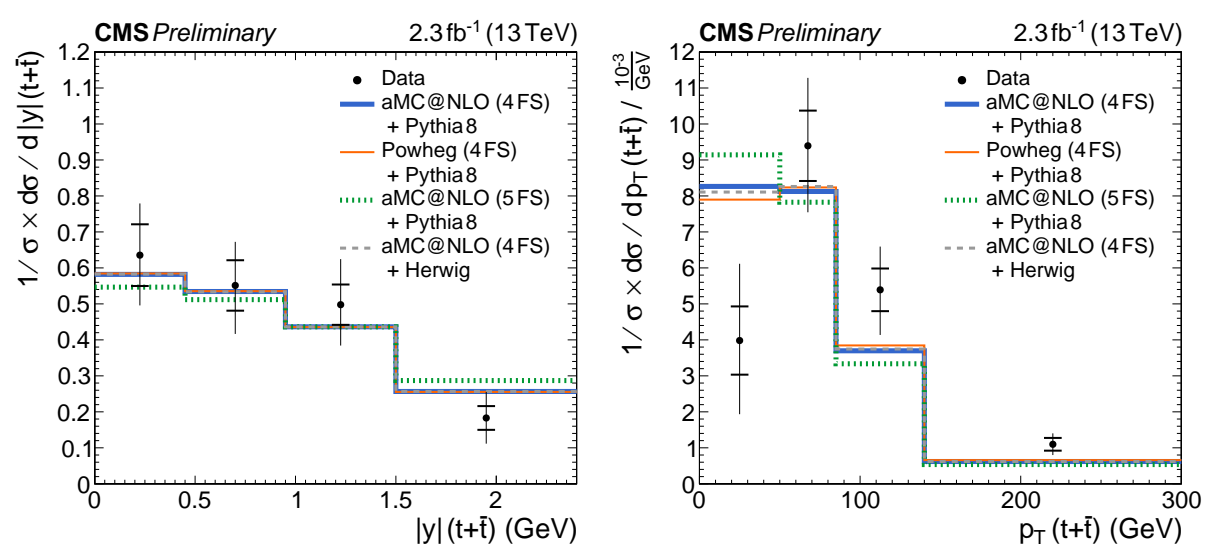

Figure 10: Measured differential $t$-channel single top quark production cross-section as a function of the top quark $p_{T}$ (left) and top quark rapidity (right) [26].

method based on BDT. The inclusive $W t$ production cross-section is measured to be $\sigma_{W t \text {-ch }}=$ $23.0 \pm 1.3$ (stat) ${ }_{-3.5}^{+3.2}$ (syst) \pm 1.1 (lumi.) pb with a significance of $7.7 \sigma$. The measured cross-section is used to extract $\left|V_{t b}\right|$ of $1.01 \pm 0.10$.

\subsection{Summary of the single top cross-section and $\left|V_{t b}\right|$ measurements}

Figure 11 shows a summary of ATLAS and CMS measurements of the single top production cross-sections and $\left|V_{t b}\right|$ in various channels and at various center of mass energies [9]. Figure 12 shows combinations of $\mathrm{CDF}$ and $\mathrm{D} \emptyset$ measurements of the single top quark production cross-sections and posterior probability density distribution for the $s+t$-channels single top quark production with the yellow region indicating the allowed values of $\left|V_{t b}\right|$ in $p \bar{p}$ collisions at $\sqrt{s}=$ $1.96 \mathrm{TeV}[10]$.

\section{Summary}

Many top quark production measurements have been provided at Tevatron and at the LHC with several center-of-mass energies $(\sqrt{s}=1.96,7,8,13 \mathrm{TeV})$ and initial states $(p \bar{p}$ and $p p)$. The latest top production results show good agreement with the SM predictions. The $p$ QCD calculations describe the top quark pair production cross-section very well in the various energies and the regions. Since precise inclusive $\sigma_{t \bar{t}}$ measurements have been achieved, the shape of the kinematical distributions, jet multiplicity, and so on becomes more important. Differential top quark pair production cross-sections have been also measured at various energies. The available MC models reproduce most shape well while indicate potential need of further tuning in certain phase-space region. The single top production cross-section measurements also show good agreements with the theoretical predictions. 

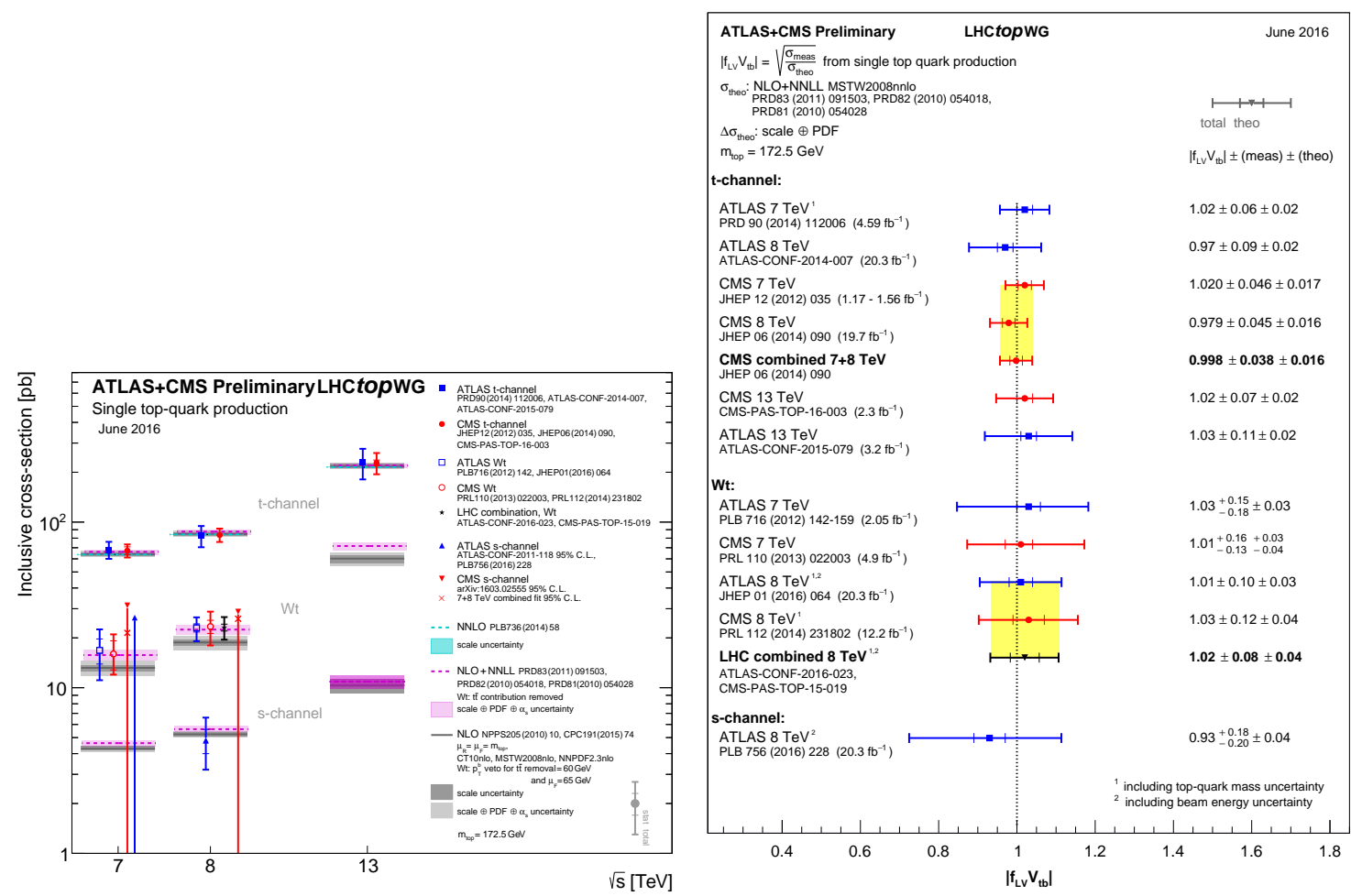

Figure 11: Summary of ATLAS and CMS measurements of the single top production cross-sections (left) and $\left|V_{t b}\right|$ (right) in various channels and at various center of mass energies [9].
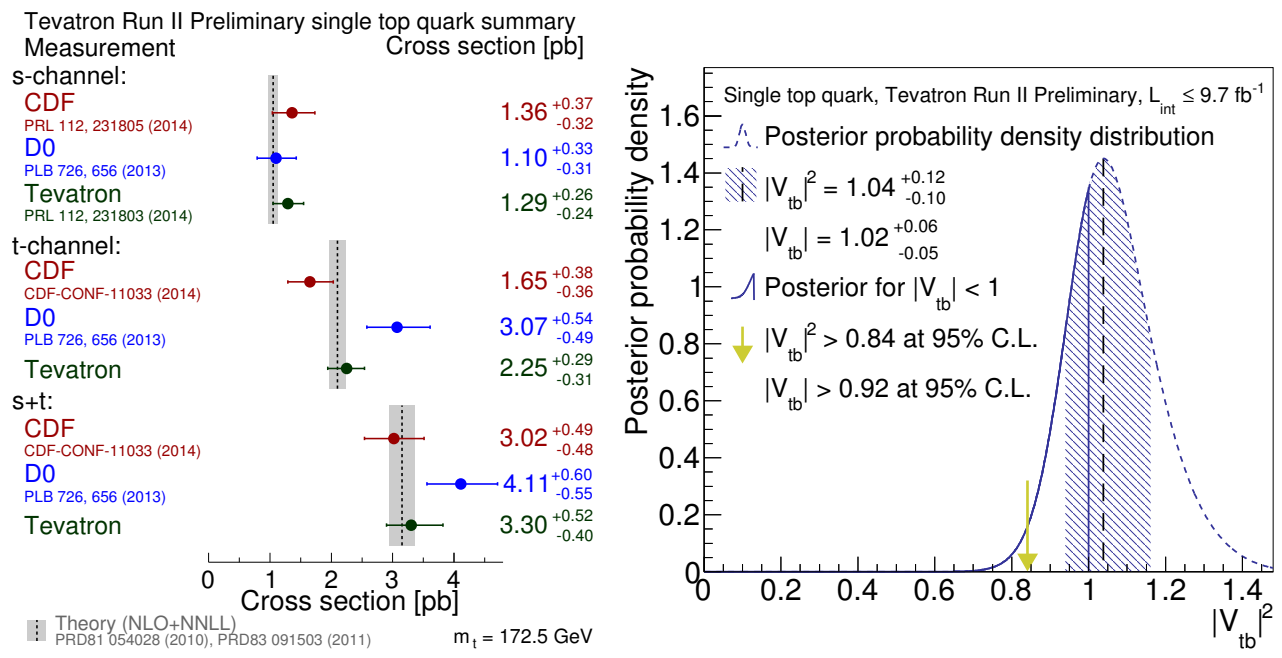

Figure 12: Summary of the all Tevatron single top cross-section measurements (left). Posterior probability density distribution for the $s+t$-channels single top quark production with the yellow region indicating the allowed values of $\left|V_{t b}\right|^{2}[10]$. 


\section{References}

[1] CDF Collaboration, Observation of top quark production in p̄p collisions, Phys. Rev. Lett. 742626 (1995) [hep-ex/9503002].

[2] DØ Collaboration, Search for high mass top quark production in p $\bar{p}$ collisions at $\sqrt{s}=1.8$ TeV, Phys. Rev. Lett. 742422 (1995) [hep-ex/9411001].

[3] CDF and D $\varnothing$ Collaborations, Combination of measurements of the top-quark pair production cross section from the Tevatron Collider, Phys. Rev. D 89072001 (2014) [hep-ex/1309 . 7570]

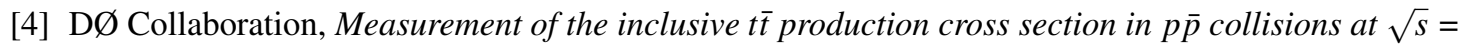
1.96 TeV, DO Note 6453-CONF (2015)

[5] CMS Collaboration, The CMS experiment at the CERN LHC, JINST 3 S08004 (2008)

[6] ATLAS Collaboration, The ATLAS Experiment at the CERN Large Hadron Collider, JINST 3 S08003 (2008)

[7] CMS Collaboration, Measurement of the top quark pair production cross section using e $\mu$ events in proton-proton collisions at $\sqrt{s}=13 \mathrm{TeV}$ with the CMS detector, CMS-PAS-TOP-16-005 (2016)

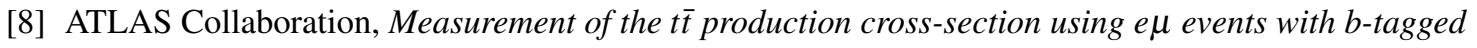
jets in pp collisions at $\sqrt{s}=13 T e V$ with the ATLAS detector, Phys. Lett. B 761136 (2016) [hep-ex/1606.02699]

[9] LHC Top Physics Working Group, LHCTopWG Summary Plots, [https://twiki.cern.ch/twiki/bin/view/LHCPhysics/LHCTopWGSummaryPlots].

[10] CDF and DØ Collaborations, Measurement of the single top quark cross section and the CKM matrix element $\left|V_{t b}\right|$ at the Tevatron, [https://www-

cdf.fnal.gov/physics/new/top/2014/stopTevCombo_webpage/stopTevCombo_webpage.html].

[11] ATLAS Collaboration, Measurements of the t $\bar{t}$ production cross-section in the dilepton and lepton-plus-jets channels and of the ratio of the $t \bar{t}$ and $Z$ boson cross-sections in pp collisions at $\sqrt{s}=$ $13 \mathrm{TeV}$ with the ATLAS detector, ATLAS-CONF-2015-049

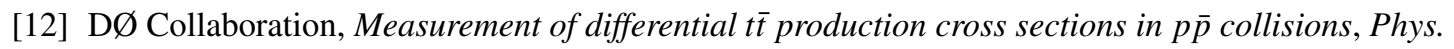
Rev. D 90092006 (2014) [hep-ex/1401.5785]

[13] CDF Collaboration, Measurement of the Differential Cross Section $d \sigma / d \cos \theta_{t}$ for Top-Quark Pair Production in p $\bar{p}$ Collisions at $\sqrt{s}=1.96$ TeV, Phys. Rev. Lett. 111182002 (2013) [hep-ex/1306.2357]

[14] ATLAS Collaboration, Measurement of jets produced in top quark events using the di-lepton final state with 2 b-tagged jets in pp collisions at $\sqrt{s}=13 \mathrm{TeV}$ with the ATLAS detector, ATLAS-CONF-2015-065 (2015)

[15] CMS Collaboration, Measurement of the inclusive and differential t $\bar{t}$ production cross sections in lepton+jets final states at $13 \mathrm{TeV}$, CMS-PAS-TOP-16-008 (2016)

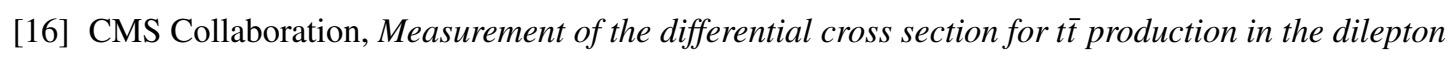
final state at $\sqrt{s}=13 \mathrm{TeV}$, CMS-PAS-TOP-16-011 (2016)

[17] ATLAS Collaboration, Measurements of top-quark pair differential cross-sections in the lepton+jets channel in pp collisions at $\sqrt{s}=8$ TeV using the ATLAS detector, Eur. Phys. J. C 76538 (2016) [hep-ex/1511.04716]. 
[18] CMS Collaboration, Measurement of the differential cross section for top quark pair production in pp collisions at $\sqrt{(s)}=8$ TeV, Eur. Phys. J. C 75542 (2015) [hep-ex/1505. 04480 ]

[19] LHCb Collaboration, The LHCb Detector at the LHC, JINST 3 S08005 (2008)

[20] LHCb Collaboration, First Observation of Top Quark Production in the Forward Region, Phys. Rev. Lett. 115112001 (2015) [hep-ex/1506.00903]

[21] CDF and DØ Collaboration, Observation of s-Channel Production of Single Top Quarks at the Tevatron, Phys. Rev. Lett. 112231803 (2014) [hep-ex/1402 . 5126]

[22] ATLAS Collaboration, Evidence for single top-quark production in the s-channel in proton-proton collisions at $\sqrt{s}=8 \mathrm{TeV}$ with the ATLAS detector using the Matrix Element Method, Phys. Lett. B 756 228-246 (2016) [hep-ex/1511.05980]

[23] CMS Collaboration, Search for s channel single top quark production in pp collisions at $\sqrt{s}=7$ and $8 \mathrm{TeV}$, Submitted to JHEP, [hep-ex/1603.02555].

[24] ATLAS Collaboration, Measurement of the inclusive cross-section of single top-quark t-channel production in pp collisions at $\sqrt{s}=13 \mathrm{TeV}$, ATLAS-CONF-2015-079 (2015)

[25] CMS Collaboration, Measurement of the inclusive cross section of single top-quark production in the t-channel at $13 \mathrm{TeV}, \mathrm{CMS}$-PAS-TOP-16-003 (2016)

[26] CMS Collaboration, Measurement of the differential cross section for $t$-channel single-top-quark production at $\sqrt{s}=13 \mathrm{TeV}$, CMS-PAS-TOP-16-004 (2016)

[27] ATLAS Collaboration, Measurement of the production cross-section of a single top quark in association with a W boson at $8 \mathrm{TeV}$ with the ATLAS experiment, JHEP 01064 (2016)

[hep-ex/1510.03752] 